\title{
Vildagliptin-induced acute pancreatitis without enzyme elevation
}

\author{
Zeynel Mungan ${ }^{1,2}$ (D), Tan Attila, ${ }^{1,2}$, Zeynep Ünal Kabaoğlu Detin Vural \\ 'Department of Gastroenterology, Koç University School of Medicine, İstanbul, Turkey \\ ${ }^{2}$ Department of Gastroenterology, VKV American Hospital, İstanbul, Turkey \\ ${ }^{3}$ Department of Radiology, VKV American Hospital, Istanbul, Turkey
}

Cite this article as: Mungan Z, Attila T, Kabaoğlu Zü, Vural M. Vildagliptin-induced acute pancreatitis without enzyme elevation. Turk J Gastroenterol 2017. 2017; 28: 514-7.

\begin{abstract}
We describe 2 patients with diabetes mellitus, presenting with upper abdominal pain. Although imaging findings were consistent with acute pancreatitis (AP), serum amylase and lipase levels were within normal limits. In both the cases, the only identifiable diagnostic culprit was vildagliptin. This is the first reported case of vildagliptin causing AP clinically and radiographically without elevated serum pancreatic enzymes. In conclusion, even when serum amylase and lipase levels are normal, AP should be kept in mind when making a differential diagnosis of patients with diabetes mellitus who present with abdominal pain and take dipeptidyl peptidase-4 (DPP-4) inhibitors.
\end{abstract}

Keywords: Pancreatitis, vildagliptin, abdominal pain, diabetes, amylase, lipase

\section{INTRODUCTION}

In Western countries, the majority of acute pancreatitis (AP) cases are caused by alcohol consumption and gallstones. The AP diagnosis requires 2 of the following 3 criteria: acute onset of severe, persistent, epigastric pain radiating to back; serum lipase or amylase levels at least 3 times greater than the upper limit of normal; signs of pancreatic inflammation or edema on imaging (1-3). Although pancreatic enzyme elevation is an important criterion for AP diagnosis, there are reports of acute pancreatitis without amylase and lipase elevations (2-5).

Dipeptidyl peptidase-4 (DPP-4) inhibitors are used to treat type 2 diabetes mellitus (DM2). Although DPP-4 inhibitors are generally considered safe, there were reports of severe pancreatitis in patients receiving sitagliptin, a DPP-4 inhibitor, which prompted the Food and Drug Administration (FDA) to issue an alert on this adverse reaction (6). A meta-analysis did not reveal an increased incidence of pancreatitis with the newer DPP-4 inhibitor, vildagliptin (7). However, there are recent reports of AP due to vildagliptin $(8,9)$. According to our knowledge, this is the first reported case of vildagliptin causing AP clinically and radiographically without serum pancreatic enzyme elevation.

\section{CASE PRESENTATIONS}

Written informed consent obtained from the patients.

\section{Case 1}

A 68-year old woman presented with a 5-day history of abdominal pain and was diagnosed with diverticulitis. She was started on cephalosporin. Her past medical history included DM2, hypertension, and diverticulosis. Vildagliptin was added to her treatment regimen 2 months prior to the admission. On physical examination, epigastric tenderness was detected. Her medications included atenolol, spironolactone, valsartan/ chlorothiazide combination, levothyroxine, gabapentin, insulin, metphormin, and vildagliptin. She denied alcohol consumption. AP was diagnosed via abdomi-

Address for Correspondence: Tan Attila E-mail: tanattila2003@yahoo.com

Received: June 1, 2017

Accepted: June 13, 2017

Available Online Date: October 25, 2017

(c) Copyright 2017 by The Turkish Society of Gastroenterology • Available online at www.turkjgastroenterol.org • DOI: 10.5152/tjg.2017.17308 

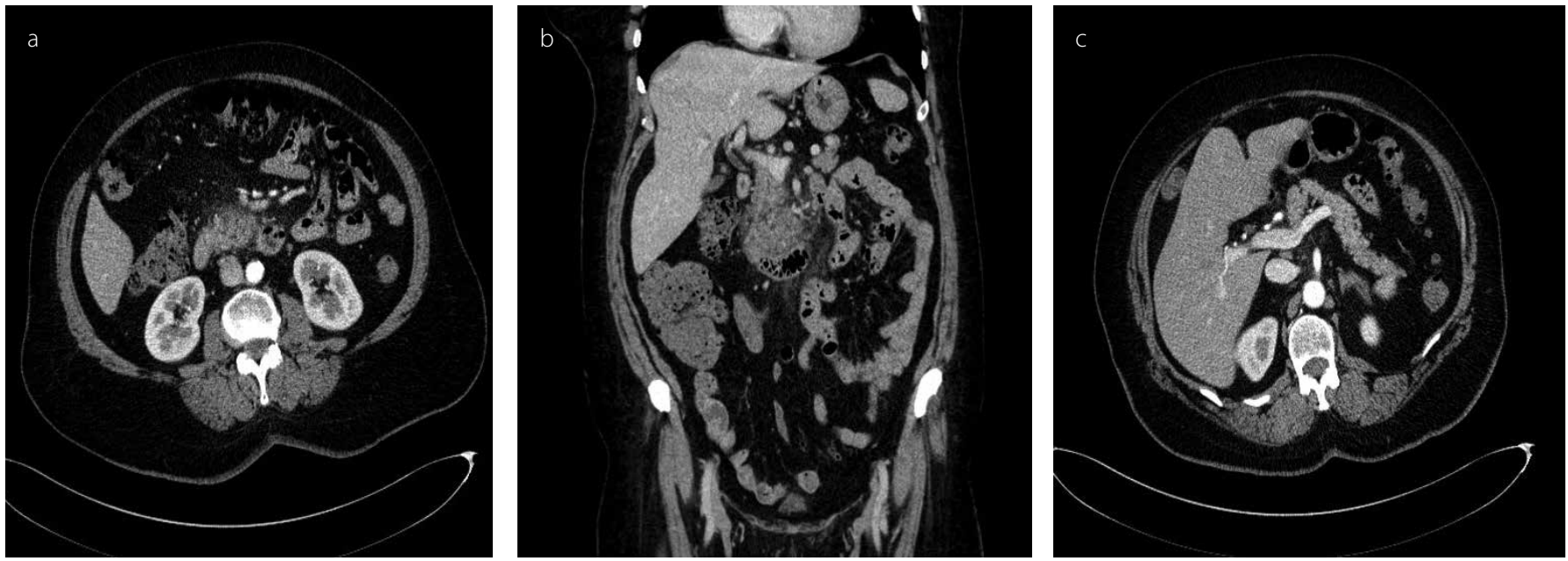

Figure 1. a-c. Axial and coronal reformatted CT images showed an enlargement and heterogeneous attenuation of the pancreatic head and uncinate process with hazy stranding of the peripancreatic fat, consistent with mild inflamatory changes $(a, b)$. The reminder of the pancreas was normal with axial image (c). There were no fluid collections, and there was no necrosis of the pancreatic parenchyma
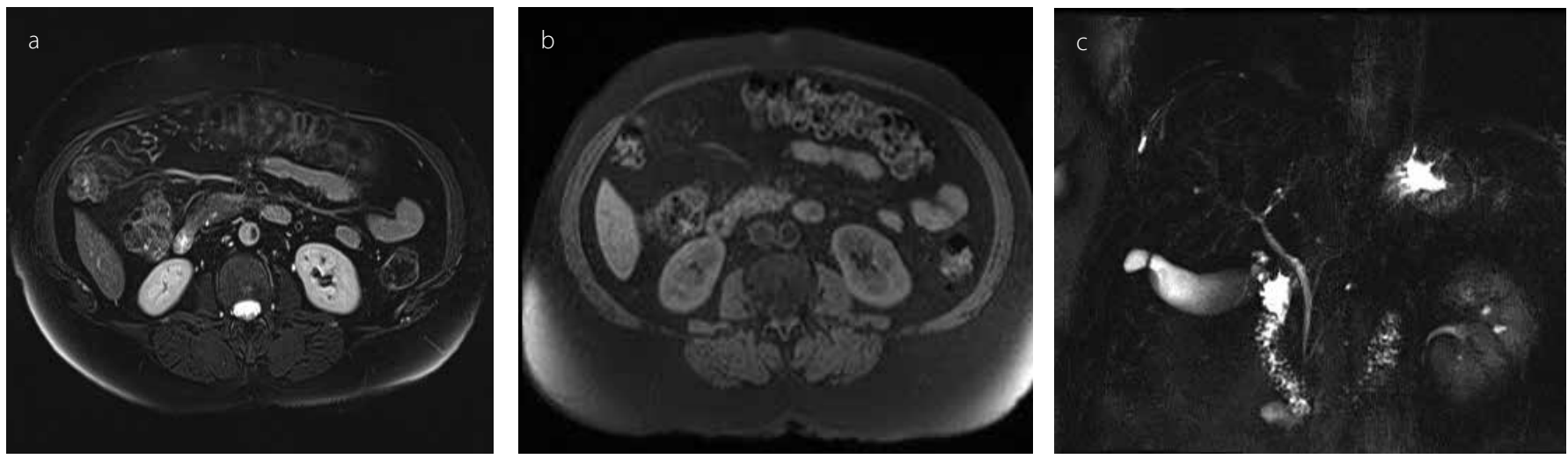

Figure 2. a-c. Axial MRI images performed 1 month later; T2-weighted with fat suppression image shows a mild hyperintens signal surrounding the pancreatic head, consistent with inflammatory changes (A). The size of pancreas (a,b) and MRCP findings (c) were normal
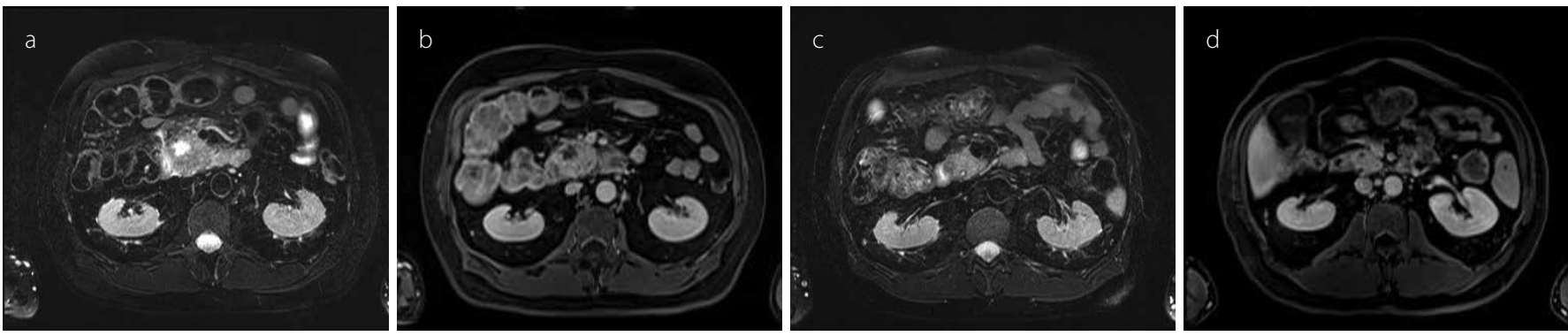

Figure 3. a-d. Axial MRI fat saturated T2- and venous phase postcontrast fat saturated T1-weighted images obtained 2 days after the onset of clinical symptoms revealed edematous swelling of proximal pancreas with small acute intrapancreatic necrotic collection and peripancreatic fat stranding (a,b). Axial MRI fat saturated T2- and venous phase postcontrast fat saturated T1-weighted images obtained 30 days after the onset of clinical symptoms revealed shrinkage of intrapancreatic necrotic collection $(c, d)$

nal computed tomography (CT; Figure 1). Laboratory tests revealed leukocytosis (16.210/mm³ normal: 4000-11000/uL) and an increased c-reactive protein (CRP) (89.3 mg/L; normal: <5 $\mathrm{mg} / \mathrm{L})$, but normal amylase (31 U/L) and lipase (50 U/L) levels. Liver findings, electrolytes, and lipids were within normal limits. The application of valsartan/chlorothiazide combination and vildagliptin was discontinued secondary to hyponatremia and $A P$, respectively. She was treated with bowel rest, hydration, and pain control. The glucose level was regulated with insulin. Oral feeding was reintroduced gradually. Amylase and lipase levels did not increase during the follow up. Leukocytes, $\mathrm{CRP}$, and sodium returned to normal levels. Control magnetic resonance (MR)/Magnetic resonance cholangiopancreatography (MRCP) within 30 days showed normal biliary system and regression of pancreatitis without any radiographic evidence of malignancy (Figure 2). The patient is still treated with valsartan+chlorothiazide, levothyroxine, gabapentin, insulin, and metformin without any recurrent abdominal pain. 


\section{Case 2}

A 52-year-old man presented with a 3-day history of severe, sharp, persistent abdominal pain, radiating to back, and nausea. His medical history indicated DM2 for 10 years, initially managed with oral anti-diabetics and then insulin for the last 2 years. Three months prior to presentation, the patient was started on vildagliptin. He did not use any other medications, and he denied alcohol consumption. On physical examination, epigastric tenderness was detected. An abdominal MR imaging revealed acute necrotizing pancreatitis (Figure 3a,b). Laboratory tests revealed leukocytosis $\left(11.760 / \mathrm{mm}^{3}\right)$ and increased CRP (37.9 mg/L), but normal amylase (31 U/L) and lipase (20 U/L). Liver findings, electrolytes, and lipids were within normal limits. The patient was treated with bowel rest, hydration, and pain control. The vildagliptin application was discontinued, and glucose level was regulated with insulin. Oral feeding was reintroduced gradually, and the patient tolerated normal diet without any symptoms within 2 weeks. Amylase and lipase levels did not increase during the follow up. Leukocytes and CRP returned to normal levels. The MRI 30 days after the acute attack showed prominent regression of pancreatic findings (Figure 3c,d). Pancreatic malignancy was excluded with MRI and normal tumor markers.

\section{DISCUSSION}

Serum amylase and lipase are used to diagnose AP. Although the amylase level is sensitive, its specificity is low. Amylase is found throughout the digestive tract and may be elevated due to numerous medical conditions, drugs, or surgical procedures. On the other hand, the amylase level can remain normal in alcohol- or hypertriglyceridemia-related AP (10). In contrast, lipase is synthesized and stored mainly within the pancreatic acinar cells; therefore, lipase is more specific than amylase for diagnosis of AP. Lipase also stays elevated for a longer time in serum, and therefore, it is a better indicator of pancreatitis in patients with delayed presentation (10). Although pancreatic enzyme elevation is an important criterion for AP diagnosis, there are reports of acute pancreatitis without amylase and lipase elevations (2-5). In our cases, clinical suspicion prompted AP diagnosis. Although pancreatic enzymes were within normal limits in both the cases, radiological evaluation revealed diagnosis. Both amylase and lipase levels were normal at the diagnosis and during the follow up. Patients denied alcohol consumption. Other causes of pancreatitis such as hypertriglyceridemia, hypercalcemia, familial history of pancreatitis, autoimmune and biliary pancreatitis, past history of pancreatitis, as well as pancreatic malignancy, were excluded. After discontinuation of vildagliptin, the patients' symptoms improved. In follow up radiologic evaluations, pancreatitis improved and no evidence of malignancy was noted. In general, it is difficult to prove that pancreatitis is clearly secondary to a specific agent. However, improvement of abdominal pain and abdominal imaging despite continuation of medications except vildagliptin supports that vildagliptin is the only possible culprit in the development of AP. DPP-4 inhibitors are important drugs for the treatment of DM2, and they are generally considered safe and well tolerated (7). An AP risk has been previously noted with this class of medications, especially with sitagliptin. However, AP is not a well-known complication of newer DPP-4 inhibitor, vildagliplin. There have been only a few AP case reports related to vildagliptin in the last few years $(8,9)$. In all these vildagliptinrelated AP cases, diagnosis was confirmed with amylase and lipase elevation and radiologic evidence of AP. According to our knowledge, this is the first reported case of vildagliptin-related acute pancreatitis without enzyme elevations.

In conclusion, even when the serum levels of amylase and lipase are normal, AP should be kept in mind when making a differential diagnosis of patients with DM who present with abdominal pain and take dipeptidyl peptidase-4 (DPP-4) inhibitors. If AP is suspected, abdominal imaging should be obtained to evaluate pancreatic parenchyma despite normal serum pancreatic enzyme levels.

Informed Consent: Written informed consent was obtained from patients who participated in this study.

Peer-review: Externally peer-reviewed.

Author contributions: Concept - Z.M., T.A.; Design - Z.M., T.A.; Supervision - Z.M.; Resource - Z.M., T.A., Z.Ü.K., M.V.; Materials - Z.M., T.A., Z.Ü.K., M.V.; Data Collection and/or Processing - Z.M., T.A., Z.Ü.K., M.V.; Analysis and/or Interpretation - Z.M., T.A.; Literature Search - Z.M., T.A.; Writing Z.M., T.A.; Critical Reviews - Z.M., T.A.

Conflict of Interest: No conflict of interest was declared by the authors.

Financial Disclosure: The authors declared that this study has received no financial support.

ORCID ID: Zeynel Mungan: 0000-0001-5952-9265

ORCID ID: Tan Attila: 0000-00026803-4632

ORCID ID: Zeynep Ünal Kabaoğlu: 0000-0002-0098-4009

ORCID ID: Metin Vural: 0000-0003-3755-2397

\section{REFERENCES}

1. Banks PA, Bollen TL, Dervenis $C$, et al. Classification of acute pancreatitis-2012: revision of the Atlanta classification and definitions by international consensus. Gut 2013; 62: 102-11. [CrossRef]

2. Cartier T, Sogni P, Perruche F, et al. Normal lipase serum level in acute pancreatitis: a case report. Emerg Med J 2006; 23:701-2. [CrossRef]

3. Shah AM, Eddi R, Kothari ST, Maksoud C, DiGiacomo S, Baddoura W. Acute pancreatitis with normal serum lipase: A case series. JOP 2010; 11: 369-72.

4. Rao EMM, Ganga V, Moore M, Medarametla S. A case of acute pancreatitis without enzyme elevation-A rare presentation of a common condition. Clin Med Rev Case Rep 2015; 2: 66. [CrossRef]

5. Mathur AK, Whitaker A, Kolli H, Nguyen T. Acute pancreatitis with normal serum lipase and amylase: A rare presentation. JOP 2016; 17: 98-101. 
6. US Food and Drug Administration. MedWatch 2009 Safety Alerts for Human products: Sitagliptin (marketed as Januvia and Janumet) - acute pancreatitis. September 25, 2009. Available at: http://www.fda.gov/Safety/MedWatch/Safetylnformation/SafetyAlertsHuman medicalProducts/ucm 183800.htm.

7. Bekiari E, Rizava C, Athanasiadou E, et al. Systematic review and meta-analysis of vildagliptin for treatment of type 2 diabetes. Endocrine 2016: 52; 458-80. [CrossRef]
8. Girgis CM, Champion BL. Vildagliptin-induced pancreatitis. Endocr Pract 2011; 17: 48-50. [CrossRef]

9. Kunjathaya P, Ramaswami PK, Krishnamurthy AN, Bhat $N$. Acute necrotizing pancreatitis associated with Vildagliptin. JOP 2013: 14; 81-4.

10. Yadav D, Agarwal N, Pitchumoni Cs. A critical evaluation of laboratory tests in acute pancreatitis. Am J Gastroenterol 2002; 97: 1309-18.[CrossRef] 